\title{
Value of Computed Tomography of the Chest in Subjects With ARDS: A Retrospective Observational Study
}

\author{
Marcel Simon MD, Stephan Braune MD, Azien Laqmani MD, Maria Metschke MD, \\ Christoph Berliner MD, Maria Kalsow, Hans Klose MD, and Stefan Kluge MD
}

\begin{abstract}
BACKGROUND: The value of computed tomography (CT) of the chest in the management of patients with ARDS is poorly defined. The aim of this study was to assess the clinical utility of thoracic CT scans in subjects with ARDS using the Berlin definition. METHODS: This was a retrospective, observational study in a university hospital ARDS center on all subjects with ARDS in whom a CT scan of the chest was performed immediately before or during an ICU stay between January 1, 2007 and June 30, 2013. RESULTS: During the study period, a total of 1,781 thoracic CT scans were performed, of which 204 cases met inclusion criteria. The most common pathologic findings of the lung parenchyma were consolidations $(94.1 \%$ of cases) and ground glass opacities (85.3\%). Furthermore, CT scans showed pleural effusions (80.4\%), mediastinal lymphadenopathy $(66.7 \%)$, signs of right ventricular strain and pulmonary hypertension $(53.9 \%)$, pericardial effusion $(37.3 \%)$, emphysema of the chest wall $(12.3 \%)$, pneumothorax $(11.8 \%)$, emphysema of the mediastinum $(7.4 \%)$, and pulmonary embolism $(2.5 \%)$. Results of CT scans led to changes in management in $26.5 \%$ of cases. Mortality was significantly increased in subjects with involvement of lung parenchyma of $>80 \%(P=.004)$. Intrahospital transport was associated with critical incidents in 8.3\% of cases. CONCLUSIONS: Systematic evaluation of thoracic CT scans yielded information useful for making a diagnosis, predicting prognosis, and recognizing concomitant disorders requiring therapeutic interventions. Results obtained from CT scans led to changes in management in 26.5\% of cases. Key words: ARDS; computed tomography; intensive care unit; intrahospital transport. [Respir Care 2016;61(3):316-323. (c) 2016 Daedalus Enterprises]
\end{abstract}

\section{Introduction}

ARDS is characterized by respiratory failure of acute onset not fully explained by cardiac failure or fluid overload and bilateral opacities in radiological imaging. ${ }^{1}$ ARDS is associated with high mortality. ${ }^{2}$

\footnotetext{
Dr Simon, Dr Braune, Dr Metschke, Ms Kalsow, and Dr Kluge are affiliated with the Department of Intensive Care Medicine, University Medical Center Hamburg-Eppendorf, Hamburg, Germany. Drs Laqmani and Berliner are affiliated with the Department of Diagnostic and Interventional Radiology, University Medical Center Hamburg-Eppendorf, Hamburg, Germany. Dr Klose is affiliated with the Department of Respiratory Medicine, University Medical Center Hamburg-Eppendorf, Hamburg, Germany.
}

The authors have disclosed no conflicts of interest.

Drs Klose and Kluge contributed equally to this work.
Whereas a conventional chest $\mathrm{x}$-ray is mandatory for the diagnosis of ARDS, computed tomography (CT) is not required to satisfy the diagnostic criteria outlined in the Berlin definition. However, CT scans in patients with ARDS are often performed for the following indications: confirmation of diagnosis, ${ }^{3}$ assessment of recruitability, ${ }^{4}$ identification of a pulmonary or extrapulmonary cause, ${ }^{5}$ prediction of prognosis, ${ }^{6-8}$ and follow-up. ${ }^{9}$ Many ARDS centers, including our department, routinely perform CT scans in patients with ARDS, believing that this may yield clinically relevant information. However, transferring critically ill patients with severe respiratory failure from the ICU for

\footnotetext{
Correspondence: Stefan Kluge MD, Department of Intensive Care Medicine, University Medical Center Hamburg-Eppendorf, Martinistr 52, 20246 Hamburg, Germany. E-mail: s.kluge@uke.de.
}

DOI: $10.4187 /$ respcare. 04308 


\section{Chest CT IN Subjects With ARDS}

$\mathrm{CT}$ is known to be a high-risk procedure and requires substantial resources regarding logistics and personnel..$^{10,11}$

Apart from studies on specific radiological aspects of ARDS, there have been few studies on the overall impact of CT on the management of patients with ARDS. ${ }^{12,13}$ The value of a CT scan and its ideal timing are poorly defined. Published studies exploring the value of CT in subjects already diagnosed with ARDS have not used the new Berlin definition and have included only small numbers of subjects. Therefore, the aim of this study was to assess the clinical utility of thoracic CT scans in patients with ARDS using the Berlin definition.

\section{Methods}

\section{Study Design}

This was a retrospective observational study conducted in the Department of Intensive Care Medicine ${ }^{14}$ at the University Medical Center Hamburg-Eppendorf, Germany. The department is an ARDS center and member of the German ARDS Network. Inclusion criteria were (1) diagnosis of ARDS using the Berlin definition ${ }^{1}$ and (2) CT scan of the chest performed on the day of ICU admission, immediately before or during ICU stay. The Ethics Committee of the Hamburg Chamber of Physicians approved the protocol and waived the need to obtain consent for the collection, analysis, and publication of the retrospectively obtained and anonymized data for this non-interventional study.

\section{Data Collection}

Data from all patients eligible for inclusion during the study period from January 1, 2007 to June 30, 2013 were collected from the electronic departmental patient data management system (Integrated Care Manager [ICM] 8.1, Dräger Medical, Lübeck, Germany). During this period, a total of 41,690 patients were treated in the Department of Intensive Care Medicine at the University Medical Center Hamburg-Eppendorf, and 1,781 thoracic CT scans were performed. Of these, 204 cases met the inclusion criteria and were analyzed in this study.

\section{CT Scans of the Chest}

In our radiology department, $\mathrm{CT}$ scans of the chest in patients with ARDS are conducted according to a standardized protocol. A high-resolution low-dose CT protocol in combination with iterative reconstruction (iDose ${ }^{4}$ ) is performed using a 256-MSCT scanner (Brilliance iCT, Philips Healthcare, Best, Netherlands) and the following parameters: collimation $128 \times 0.625 \mathrm{~mm}$, tube voltage $120 \mathrm{kV}$, current-time product $20-40 \mathrm{mAs}$, pitch 0.993 ,

\section{QUICK LOOK}

\section{Current knowledge}

The value of computed tomography (CT) scans in the management of patients with ARDS is poorly defined. Published studies exploring the value of CT in subjects already diagnosed with ARDS have not used the new Berlin definition and have included only small numbers of subjects.

\section{What this paper contributes to our knowledge}

This retrospective study showed that in critically ill subjects with ARDS, thoracic CT scans provide clinicians with a comprehensive evaluation of lung parenchyma, pleural space, heart and thoracic vessels, soft tissue, and mediastinum as well as the position of invasive devices. This information is useful for confirming or changing a diagnosis and recognizing concomitant disorders requiring therapeutic interventions. Intrahospital transport for CT scans can be performed relatively safely.

slice thickness $1 \mathrm{~mm}$, increment $0.5 \mathrm{~mm}$. For special clinical indications such as pulmonary embolism and due to technical advancements during the study period, another CT scanner (Brilliance 64, Philips Healthcare, Best, Netherlands) and different protocols (collimation $64 \times 0.625$ or $128 \times 0.625 \mathrm{~mm}$, tube voltage $120 \mathrm{kV}$, current-time product 120-60 mAs, pitch 1.078-0.993) were also used. If required for the assessment of thoracic vessels, mediastinal masses, or empyema, intravenous contrast material (70 mL, Imeron 300, Bracco Altana Pharma, Milan, Italy) was administered via power injector (Medrad, Stellant D, Bayer HealthCare, Whippany, New Jersey) at a flow rate of $2 \mathrm{~mL} / \mathrm{s}$. Images were obtained with a post-injection delay of $40 \mathrm{~s}$. For the assessment of pulmonary embolism, intravenous bolus injection of contrast material $(70 \mathrm{~mL}$, Imeron 400, Bracco Altana Pharma, Milan, Italy) was administered at a rate of $4 \mathrm{~mL} / \mathrm{s}$ through an 18-gauge peripheral venous catheter. For contrast optimization of pulmonary arteries, a bolus tracking technique was used with a data acquisition start delay of $4 \mathrm{~s}$ after exceeding a threshold level of 130 Hounsfield units within the main pulmonary artery.

\section{Evaluation of CT Scans}

Two experienced radiologists (AL and CB) who had no knowledge of the subjects' history re-evaluated all CT scans for study purposes in consensus. The findings were described using published nomenclature for thoracic im- 


\section{Chest CT in Subjects With ARDS}

aging. ${ }^{15}$ Data were collected concerning pathologic findings of lung parenchyma, pleural space, heart and thoracic vessels, soft tissue, and mediastinum as well as devices.

\section{Evaluation of Lung Parenchyma}

Pathologic findings of lung parenchyma were defined as the presence of atelectasis, bronchiectasis, cavities, consolidations, emphysema, ground glass opacities, honeycombing, interstitial changes, masses, or tree-in-bud pattern. ${ }^{16}$ The percentage of lung involvement was estimated qualitatively to the nearest 10th percentile (eg, 10\%, 20\%) by evaluating the axial and coronal CT images. ${ }^{6}$ The radiologist suggested a specific etiology when a characteristic pattern was identified on CT scans.

\section{Evaluation of the Pleural Space}

The presence of fluid or air within the pleural space was recorded. Both findings were categorized according to their size. Pleural effusions were measured by assessing the maximum perpendicular diameter to the parietal pleura at the greatest depth on axial CT images. ${ }^{17}$ A pleural effusion was categorized as small if its diameter was $<2 \mathrm{~cm}$, medium size if its diameter was $\geq 2 \mathrm{~cm}$ but $<5 \mathrm{~cm}$, and large if its diameter was $\geq 5 \mathrm{~cm}$. Pneumothorax was graded into 3 categories using a modified CT classification by assessing the anteroposterior diameter on axial CT images and the craniocaudal diameter ${ }^{18}$ : (1) small if its anteroposterior diameter on the axial plane was $<1 \mathrm{~cm}$ and its craniocaudal diameter was $<4 \mathrm{~cm}$, (2) medium if its anteroposterior diameter was $\geq 1 \mathrm{~cm}$ but did not extend across the midcoronal line and its craniocaudal diameter was $<4 \mathrm{~cm}$, and (3) large if its anteroposterior diameter extended across the mid-coronal line or its craniocaudal diameter was $\geq 4 \mathrm{~cm}$.

\section{Evaluation of Heart and Thoracic Vessels}

Heart and thoracic vessels were assessed on post-contrast CT scans. Signs of right ventricular strain and pulmonary hypertension were defined as a diameter of the pulmonary artery $>3 \mathrm{~cm}$, a ratio of right to left atrial diameter of $>1$, or reflux of intravenous contrast into the inferior vena cava. ${ }^{19}$ Pulmonary arteries were assessed for emboli. The presence of a pericardial effusion and its diameter were also recorded.

\section{Evaluation of Soft Tissue and Mediastinum}

Soft tissue and mediastinum were evaluated for the presence of air, masses, and lymphadenopathy.

\section{Evaluation of Devices}

The presence and correct position of endotracheal tubes, central venous catheters, and chest tubes were recorded. An endotracheal tube was defined to be misplaced or in a suboptimal position if its tip was found outside the trachea, if it reached into the right or left main bronchus, or if it was in contact with the main carina. A central venous catheter was defined to be misplaced or in a suboptimal position if its tip did not reach the superior vena cava. A chest tube was defined to be malpositioned if it did not reach the pleural space or if it was placed within or running through lung parenchyma.

\section{Complications Associated With Intrahospital Transport}

Electronic medical records were screened for documented critical incidents during or related to intrahospital transport. Dislocation of catheters or tubes, malfunction of devices, any respiratory worsening, and/or any event complicating or leading to the prolongation or abortion of the radiological examination were considered adverse events. Respiratory worsening was defined as a decrease in oxygen saturation of $10 \%$ or more or an increase in $\mathrm{P}_{\mathrm{aCO}_{2}}$ of $10 \mathrm{~mm} \mathrm{Hg}$ or more from baseline.

\section{Treatment Changes and Outcome}

Two authors (MS and MK) reviewed all medical records. Treatment changes related to the results of the CT scan were retrieved, including changes of medication and any related interventions or surgery. Data concerning length of ICU stay, length of hospital stay, ICU mortality, and mortality at $28 \mathrm{~d}$ after ICU admission were also collected.

\section{Data Analysis}

Continuous variables are presented as medians and ranges, and categorical variables are presented as absolute numbers and percentages. For univariate subgroup analysis of mortality, the chi-square test was used. A 2-sided $P$ value of $<.05$ was considered significant. The software used for statistical analyses was SPSS 20.0 (SPSS, Chicago, Illinois).

\section{Results}

\section{Characteristics of Subjects}

We included 204 subjects in this study. Characteristics of subjects are shown in Table 1. Clinical features of ARDS are shown in Table 2. 


\section{Chest CT in Subjects With ARDS}

Table 1. Characteristics of Subjects

\begin{tabular}{|c|c|}
\hline Characteristics & Values \\
\hline Subjects, N & 204 \\
\hline Age, median (range) y & $55(16-87)$ \\
\hline \multicolumn{2}{|l|}{ Sex, $n(\%)$} \\
\hline Male & $138(67.6)$ \\
\hline Female & $66(32.4)$ \\
\hline SAPS II on ICU admission, median (range) & $43(13-86)$ \\
\hline \multicolumn{2}{|l|}{ Main diagnosis, $n(\%)$} \\
\hline Medical & $154(75.5)$ \\
\hline Surgical & $41(20.1)$ \\
\hline Neurological & $9(4.4)$ \\
\hline \multicolumn{2}{|l|}{ Mode of admittance, $n(\%)$} \\
\hline From regular hospital ward & $94(46.1)$ \\
\hline From other hospital & $86(42.2)$ \\
\hline Via emergency department & $24(11.8)$ \\
\hline $\begin{array}{l}\text { Duration of hospitalization prior to ICU admission, } \\
\text { median (range) d }\end{array}$ & $4(0-106)$ \\
\hline \multicolumn{2}{|l|}{ Ventilation, $n(\%)$} \\
\hline Invasive ventilation & $199(97.5)$ \\
\hline Noninvasive ventilation & $5(2.5)$ \\
\hline \multicolumn{2}{|l|}{ Extracorporeal assist devices, $n(\%)$} \\
\hline High-flow VV ECMO & $45(22.1)$ \\
\hline PECLA or low-flow VV ECMO & $39(19.2)$ \\
\hline VA ECMO & $2(1.0)$ \\
\hline \multicolumn{2}{|l|}{ Additional treatment, $n(\%)$} \\
\hline Inhaled nitric oxide & $80(39.2)$ \\
\hline Prone positioning & $67(32.8)$ \\
\hline High-frequency oscillatory ventilation & $3(1.5)$ \\
\hline \multicolumn{2}{|l|}{$\begin{array}{l}\text { SAPS }=\text { Simplified Acute Physiology Score } \\
\text { ECMO }=\text { extracorporeal membrane oxygenation } \\
\text { VV ECMO = venovenous ECMO } \\
\text { PECLA = pumpless extracorporeal lung assist } \\
\text { VA ECMO = venoarterial ECMO }\end{array}$} \\
\hline
\end{tabular}

\section{CT Scans}

CT scans were performed immediately before ICU admission in $12.3 \%$ of cases. In all other cases, CT scans were performed at a median of $5 \mathrm{~d}$ (range 1-48 d) after ICU admission. During intrahospital transport and CT scanning, $80.4 \%$ of subjects were on invasive mechanical ventilation, and $3.9 \%$ were on noninvasive ventilation. $15.7 \%$ of subjects received oxygen via face mask or high-flow nasal cannula but were transferred to noninvasive ventilation immediately after CT scanning. Additionally, 6.9\% of subjects were on extracorporeal lung support. Intravenous contrast medium allowing the assessment of heart and thoracic vessels was used in $74.5 \%$ of cases.

Pathologic findings of lung parenchyma (Fig. 1) involved a median of $80 \%$ (range 5-100) of the total lung parenchyma. More than $80 \%$ of total lung parenchyma was involved in $59.3 \%$ of cases. Most often, consolidations $(94.1 \%)$ and ground glass opacities $(85.3 \%)$ were
Table 2. Clinical Features of ARDS

\begin{tabular}{|c|c|}
\hline Features & Values \\
\hline \multicolumn{2}{|l|}{ Onset, $n(\%)$} \\
\hline Present at hospital admission & $69(33.8)$ \\
\hline Developed during hospital stay & $135(66.2)$ \\
\hline Primary ARDS, $n(\%)$ & $197(96.6)$ \\
\hline Hospital-acquired pneumonia & $110(53.9)$ \\
\hline Community-acquired pneumonia & $67(32.8)$ \\
\hline Interstitial lung disease & $12(5.9)$ \\
\hline Drug-induced & 5 \\
\hline Radiotherapy-induced & 2 \\
\hline Non-specific interstitial pneumonia & 1 \\
\hline Sarcoidosis & 1 \\
\hline Other & 3 \\
\hline Aspiration & $4(2.0)$ \\
\hline Tuberculosis & $2(1.0)$ \\
\hline Alveolar hemorrhage & $1(0.5)$ \\
\hline Toxic origin & $1(0.5)$ \\
\hline Secondary ARDS, $n(\%)$ & $7(3.4)$ \\
\hline Sepsis & $7(3.4)$ \\
\hline \multicolumn{2}{|l|}{ Severity, $n(\%)$} \\
\hline Severe $\left(\mathrm{P}_{\mathrm{aO}_{2}} / \mathrm{F}_{\mathrm{IO}_{2}} \leq 100 \mathrm{~mm} \mathrm{Hg}\right)$ & $172(84.3)$ \\
\hline Moderate $\left(\mathrm{P}_{\mathrm{aO}_{2}} / \mathrm{F}_{\mathrm{IO}_{2}} 101-200 \mathrm{~mm} \mathrm{Hg}\right)$ & $31(15.2)$ \\
\hline Mild $\left(\mathrm{P}_{\mathrm{aO}_{2}} / \mathrm{F}_{\mathrm{IO}_{2}} 201-300 \mathrm{~mm} \mathrm{Hg}\right)$ & $1(0.5)$ \\
\hline $\mathrm{P}_{\mathrm{aO}_{2}} / \mathrm{F}_{\mathrm{IO}_{2}}$, median (range) $\mathrm{mm} \mathrm{Hg}$ & $66(30-208)$ \\
\hline $\mathrm{P}_{\mathrm{aCO}_{2}}>45 \mathrm{~mm} \mathrm{Hg}, n(\%)$ & $162(79.4)$ \\
\hline
\end{tabular}

identified. In $95.6 \%$ of cases, more than one pathologic pattern was found simultaneously. The patterns prompted the radiologist to suspect a specific pathogen in $20.1 \%$ of cases. Pneumocystis pneumonia was suspected in 7 cases (3.4\%) and was confirmed microbiologically in 2 cases. Aspergillus infection was presumed in 34 cases (16.7\%) and confirmed in 28 cases. $80.4 \%$ of subjects were diagnosed with pleural effusions and $11.8 \%$ with pneumothorax (Fig. 2). Pulmonary emboli were detected in 5 cases (2.5\%), of which 4 were newly diagnosed. Signs of right ventricular strain and pulmonary hypertension were found in 53.9\% of cases (Fig. 3). Pericardial effusions were seen in $37.3 \%$ of cases. Emphysema of the chest wall was found in $12.3 \%$ of cases. It was most often $(76.0 \%)$ associated with a pneumothorax or the presence of a chest tube. For details on the results of CT scans, see Table 3.

\section{Treatment Changes}

Results of CT scans led to 69 changes in management in 54 cases $(26.5 \%)$. More than one change was performed in 15 cases $(7.4 \%)$. The most common changes were alterations in antibiotic therapy ( $8.3 \%$ of cases), drainage of pleural effusions $(7.8 \%)$, and alterations in antimycotic therapy (4.4\%). Details concerning the modifications of therapeutic regimes are summarized in Table 4. 


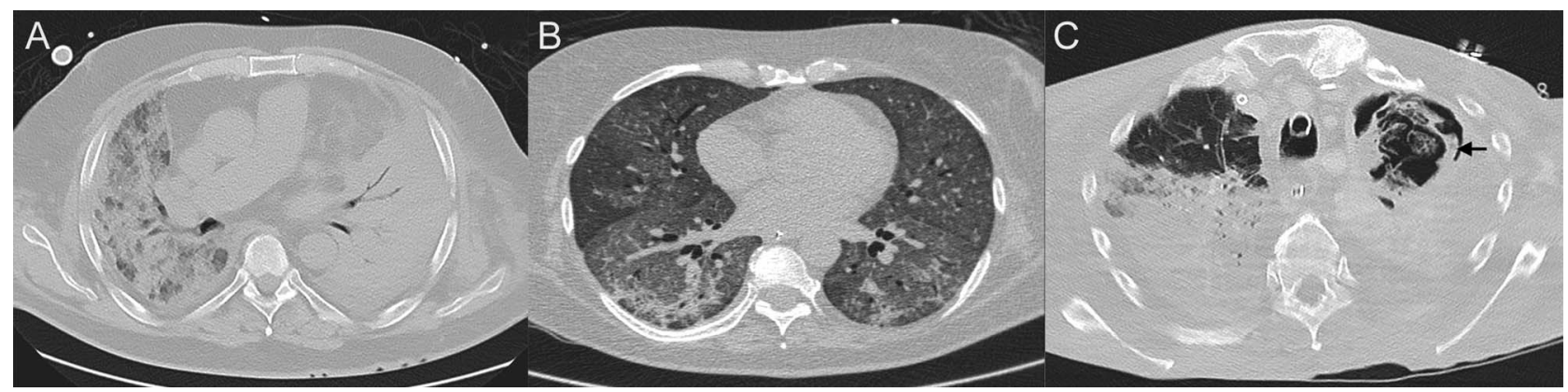

Fig. 1. Axial computed tomography images without contrast enhancement of 3 different subjects affected by community-acquired pneumonia (A), pneumocystis pneumonia (B), and aspergillosis, indicated by an arrow (C), respectively.
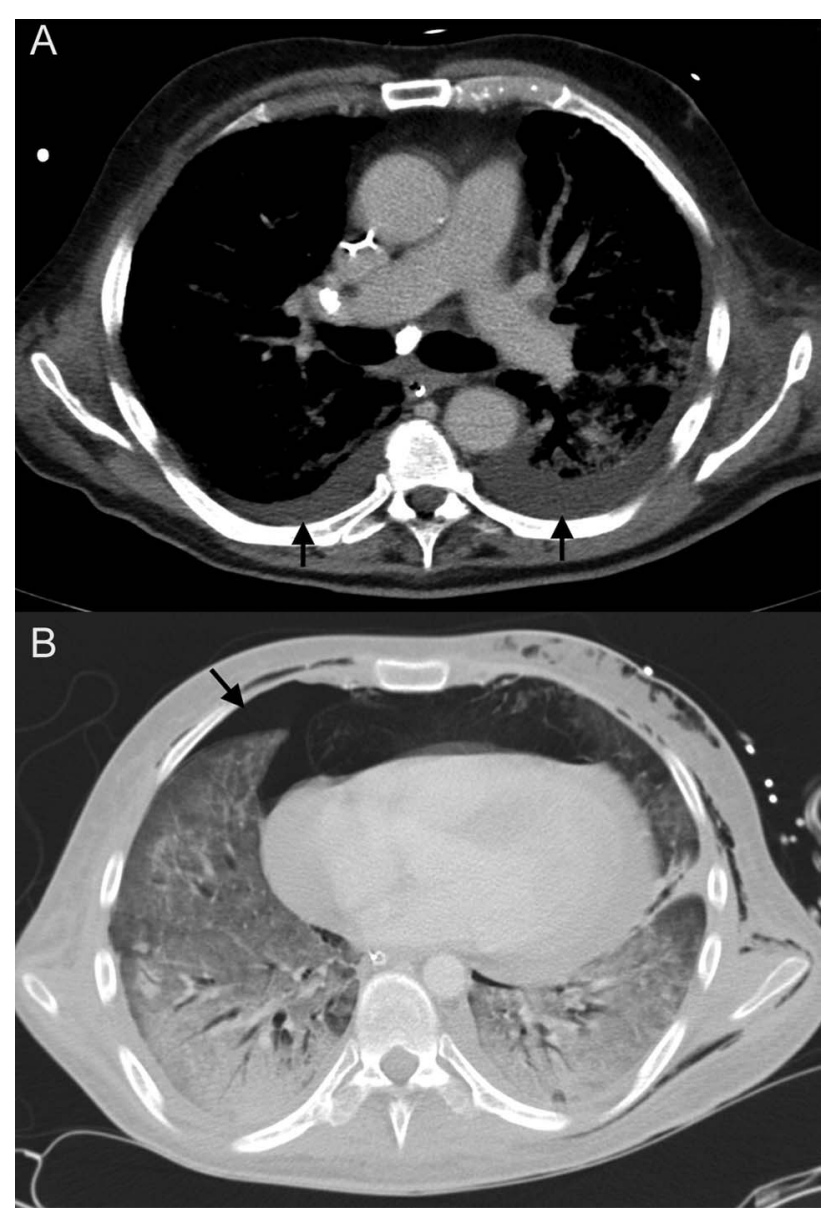

Fig. 2. Contrast-enhanced axial computed tomography images of 2 different subjects showing pleural effusion (A) and pneumotho$\operatorname{rax}(\mathrm{B})$, indicated by arrows.

\section{Outcome}

Median stay in ICU and hospital were $22 \mathrm{~d}$ (range 1-244 d) and $27 \mathrm{~d}$ (range 1-244 d), respectively. ICU and hospital mortality rates were 54.4 and $57.8 \%$, respectively. Mortality was significantly higher in subjects with pathologic involvement of their lung parenchyma of $>80 \%$
$(P=.004)$. There was no correlation between the number of parenchymal patterns and mortality $(P=.79)$. Subjects exhibiting signs of right ventricular strain and pulmonary hypertension were significantly more likely to die if dilatation of the pulmonary artery exceeded $3 \mathrm{~cm}(P=.02)$. We observed a trend in the correlation between mortality and the ratio of right to left atrial diameter of $>1(P=.08)$. Subjects were significantly less likely to die if the results of the CT scan led to changes in management $(P=.02)$. There was no difference in survival if $\mathrm{CT}$ was performed within $1 \mathrm{~d}$ of ICU admission compared with CTs performed later during the ICU stay.

\section{Complications of Intrahospital Transport}

Moving the subjects from the ICU to the radiology department led to critical incidents in $8.3 \%$ of cases: 2 subjects experienced worsening hypoxemia, and 14 subjects experienced deterioration of ventilation with subsequent hypercapnia. Two subjects deteriorated hemodynamically. In one case, the endotracheal tube dislocated when transferring the subject from the ICU bed to the CT scanner table. The endotracheal tube could be repositioned, and after completion of the CT scan, the subject returned to the ICU without further incident. All subjects fully recovered to their baseline levels within a few hours after return to the ICU. None of these incidents prevented the performance or the completion of the $\mathrm{CT}$ scan.

\section{Discussion}

Using the Berlin definition, we analyzed 204 subjects with ARDS in whom a CT scan of the chest was performed immediately before or during ICU stay. In $26.5 \%$ of cases, results obtained from thoracic CT scans led to changes in management. Critical incidents associated with intrahospital transport were observed in $8.3 \%$ of cases.

In ICU patients, CT scanning has been shown to be a valuable diagnostic tool. A recently published prospective study ${ }^{20}$ evaluating the diagnostic yield and safety of CT 


\section{Chest CT In Subjects With ARDS}

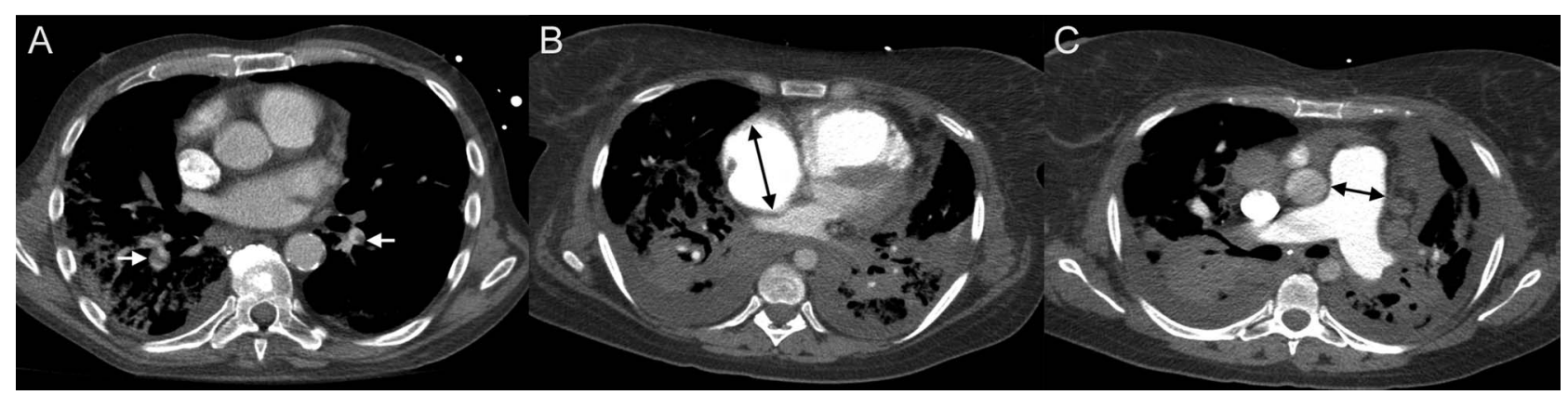

Fig. 3. Contrast-enhanced axial computed tomography images of a subject showing pulmonary emboli (A) and of another subject showing enlargement of the right atrium (B) and dilatation of the pulmonary artery (C), indicated by arrows.

scans in critically ill subjects showed that new elements were introduced to the diagnoses in $22.9 \%$ of cases. Apart from studies on specific radiological aspects of CT scans, there is only a limited number of older publications assessing the overall value of chest $\mathrm{CT}$ scans with regard to therapeutic changes in critically ill subjects with ARDS. A study by Tagliabue et al ${ }^{12}$ published in 1994 investigated 74 subjects with ARDS and found that additional information from CT scans was obtained in $66 \%$ of cases and led to treatment changes in $22 \%$ of cases. Another study on 85 ICU subjects with chest CT scans published in 1998 revealed that clinically important findings were detected in $30 \%$ of cases and led to changes in management in $22 \%$ of cases. ${ }^{13}$ In line with these results, we recorded treatment changes based on the results of the CT scans in $26.5 \%$ of subjects with ARDS according to the Berlin definition.

Previous studies have shown that intrahospital transport of critically ill subjects may be associated with significant adverse events. A study on 3,006 intrahospital transports of 1,782 subjects on invasive mechanical ventilation found a complication rate of 37.4\%.${ }^{11}$ Another study by Parmentier-Decrucq et al ${ }^{10}$ on 262 intrahospital transports reported adverse events in $45.8 \%$ and serious complications in $16.8 \%$ of cases. However, these adverse events did not lead to an increased length of time on mechanical ventilation or prolonged ICU stay. ${ }^{10}$ In their recently published study on CT scans in critically ill subjects, Aliaga et al ${ }^{20}$ recorded adverse events associated with intrahospital transport in $22.3 \%$ of cases. The event rate was significantly higher when CT scans were performed within the first $48 \mathrm{~h}$ of ICU admission $(P=.02) .{ }^{20} \mathrm{~A}$ systematic review summarizing studies on intrahospital transport of critically ill subjects concluded that optimal equipment, the use of intrahospital transport checklists, and adequate staff training were effective means to increase the safety of intrahospital transport. ${ }^{21}$ In our study, $8.3 \%$ of subjects experienced adverse events that led to transient deterioration in their condition but resolved within a few hours. It should be noted that in our department, intrahospital transport is performed according to standardized departmental protocol.
In line with our findings, Chung et $\mathrm{al}^{6}$ found that parenchymal involvement of $>80 \%$ shown on CT scans of subjects with ARDS was associated with high mortality. The study by Chung et $\mathrm{al}^{6}$ defining CT predictors of mortality in subjects with ARDS also demonstrated that a ratio of right to left atrial diameter of $>1$ was significantly more common in non-survivors than in survivors. Dilatation of the pulmonary artery of $>3 \mathrm{~cm}$ was also associated with higher mortality; however, this trend did not reach statistical significance. ${ }^{6}$ In our study, a ratio of right to left atrial diameter of $>1$ and reflux of intravenous contrast into the inferior vena cava were also associated with increased mortality, but these trends did not reach statistical significance. However, dilatation of the pulmonary artery of $>3 \mathrm{~cm}$ was found significantly more frequently in nonsurvivors than in survivors. Dilatation of the pulmonary artery seen on CT scan has been shown previously to be an independent risk factor for death in subjects with pulmonary arterial hypertension and inoperable chronic thromboembolic pulmonary hypertension. ${ }^{22}$ However, it should be noted that the evaluation of heart and thoracic vessels is feasible only in CT scans performed with the use of intravenous contrast. In our study, intravenous contrast was administered in $74.5 \%$ of cases. In the recently published study by Aliaga et al, ${ }^{20}$ intravenous contrast was used in $82 \%$ of cases. In the earlier study by Miller et al, ${ }^{13}$ intravenous contrast was used in only $57 \%$ of cases, and no intravenous contrast was used in the study by Tagliabue et al. ${ }^{12}$

The role of CT in diagnosing specific pathogens and discriminating infectious pneumonia from noninfectious diseases has been summarized in a review by Nambu et al. ${ }^{23}$ The authors of the review conclude that although CT is sometimes suggestive of specific pathogens and can offer clues to the differentiation between infectious pneumonia and noninfectious diseases, imaging findings are varied and often nonspecific. In line with this statement, in our study, the radiologist determined radiologic patterns typical for a specific pathogen in only $20.1 \%$ of cases. However, in $73.2 \%$ of these cases, the suspected pathogen could then be confirmed microbiologically. 


\section{Chest CT IN Subjects With ARDS}

Table 3. Results of Computed Tomography Scans

\begin{tabular}{|c|c|}
\hline Findings Analyzed by Computed Tomography Scans & $n(\%)$ \\
\hline \multicolumn{2}{|l|}{ Lung parenchyma } \\
\hline Consolidations & $192(94.1)$ \\
\hline Ground glass opacities & $174(85.3)$ \\
\hline Interstitial changes & $121(59.3)$ \\
\hline Atelectasis & $97(47.5)$ \\
\hline Bronchiectasis & $83(40.7)$ \\
\hline Emphysema & $52(25.5)$ \\
\hline Tree-in-bud pattern & $23(11.3)$ \\
\hline Honeycombing & $18(8.8)$ \\
\hline Cavities & $11(5.4)$ \\
\hline Masses & $11(5.4)$ \\
\hline \multicolumn{2}{|l|}{ Number of patterns found simultaneously } \\
\hline 1 pattern & $9(4.4)$ \\
\hline 2 patterns & $30(14.7)$ \\
\hline 3 patterns & $55(27.0)$ \\
\hline 4 patterns & $43(22.1)$ \\
\hline 5 patterns & $39(19.1)$ \\
\hline 6 patterns & $18(8.8)$ \\
\hline$>6$ patterns & $10(4.9)$ \\
\hline \multicolumn{2}{|l|}{ Pleural space } \\
\hline Pleural effusion & $164(80.4)$ \\
\hline Small & $73(44.5)$ \\
\hline Medium & $69(42.1)$ \\
\hline Large & $22(13.4)$ \\
\hline Pneumothorax & $24(11.8)$ \\
\hline Small & $12(50.0)$ \\
\hline Medium & $8(33.3)$ \\
\hline Large & $4(16.7)$ \\
\hline \multicolumn{2}{|l|}{ Heart and thoracic vessels* } \\
\hline $\begin{array}{l}\text { Signs of right-ventricular strain and pulmonary } \\
\text { hypertension }\end{array}$ & $110(53.9)$ \\
\hline Pulmonary arterial diameter $>3 \mathrm{~cm}$ & $81(73.6)$ \\
\hline Right atrial diameter $>$ left atrial diameter & $53(48.2)$ \\
\hline $\begin{array}{l}\text { Reflux of intravenous contrast into the inferior } \\
\text { vena cava }\end{array}$ & $19(17.3)$ \\
\hline Pericardial effusion & $76(37.3)$ \\
\hline Pulmonary embolism & $5(2.5)$ \\
\hline \multicolumn{2}{|l|}{ Soft tissue and mediastinum } \\
\hline Mediastinal lymphadenopathy & $136(66.7)$ \\
\hline Emphysema of the chest wall & $25(12.3)$ \\
\hline Emphysema of the mediastinum & $15(7.4)$ \\
\hline \multicolumn{2}{|l|}{ Devices } \\
\hline Presence of central venous catheter & $169(82.8)$ \\
\hline Suboptimal position & $9(5.3)$ \\
\hline Presence of endotracheal tube & $164(80.4)$ \\
\hline Misplaced or suboptimal position & $9(5.5)$ \\
\hline Presence of chest tube & $38(18.6)$ \\
\hline Malpositioned & $2(5.3)$ \\
\hline
\end{tabular}

It is remarkable that in our study, $13.4 \%$ of subjects were diagnosed with large pleural effusions, and $16.7 \%$ were diagnosed with large pneumothorax. It has been shown
Table 4. Treatment Changes

\begin{tabular}{lr}
\hline \hline \multicolumn{1}{c}{ Treatment Changes } & $n(\%)$ \\
\hline Changes in antibiotic therapy & $17(8.3)$ \\
Drainage of pleural effusion & $16(7.8)$ \\
Changes in antimycotic therapy & $9(4.4)$ \\
Correction of position of endotracheal tube & $7(3.4)$ \\
Measures to achieve negative fluid balance & $6(2.9)$ \\
Institution of therapeutic anticoagulation & $5(2.5)$ \\
Correction of position of chest tube & $2(1.0)$ \\
Prone positioning & $2(1.0)$ \\
Correction of position of central venous catheter & $1(0.5)$ \\
Drainage of pneumothorax & $1(0.5)$ \\
Institution of therapy with systemic steroids & $1(0.5)$ \\
Systemic thrombolysis & $1(0.5)$ \\
Treatment for coagulopathy & $1(0.5)$ \\
Total changes in treatment & $69(100)$ \\
\hline
\end{tabular}

previously that CT scans may reveal additional pathologic findings of the pleural space compared with conventional chest radiographs. In the study by Miller et al ${ }^{13}$ of 108 thoracic CT scans, 94 small pleural effusions and 14 small pneumothoraces as well as 2 large pleural effusions and one large pneumothorax considered clinically important findings were newly diagnosed. However, in the evaluation of the pleural space, ultrasound is a valuable diagnostic tool and may substitute for CT scans in selected cases.

There is much debate on the optimal use of imaging techniques, including CT, ultrasound, positron emission tomography, electrical impedance tomography, and magnetic resonance imaging in patients with ARDS. ${ }^{24-26} \mathrm{Sev}$ eral aspects, such as the underlying diagnostic question, bedside availability, radiation exposure, and inter-observer variability need to be taken into account for each case and setting to select the most appropriate imaging modality. Recently, the use of lung ultrasound, which is noninvasive and available at the bedside, has increasingly been propagated in critically ill patients, ${ }^{27}$ including those with ARDS. ${ }^{28}$ However, chest CT scans offer the advantage of a comprehensive approach independent of user skills not only for the evaluation of lung parenchyma, but also for the assessment of pleural space, heart and thoracic vessels, soft tissue, and mediastinum as well as the position of catheters and tubes.

Our study has some methodological limitations. The interpretation of the results is limited by potential biases introduced by the retrospective study design. Minor complications during intrahospital transport may have been missed due to incomplete documentation. However, we believe that because of their clinical relevance, all severe and life-threatening incidents were fully documented at the time of their occurrence and therefore were not missed by retrospective analysis. Furthermore, our results are only applicable to patients with similar characteristics. It is therefore im- 


\section{Chest CT IN Subjects With ARDS}

portant to note that most critically ill subjects in this study had moderate or severe ARDS, and more than one third of subjects needed extracorporeal lung support. Finally, when generalizing the results to other settings, it must be remembered that although subjects were treated as part of routine clinical care, this study was conducted in a center that is highly experienced in the treatment of patients with ARDS and in the use of extracorporeal lung support.

\section{Conclusions}

In critically ill patients with ARDS, defined by the criteria of the Berlin definition, CT scan of the chest provides clinicians with a comprehensive evaluation of lung parenchyma, pleural space, heart and thoracic vessels, soft tissue, and mediastinum as well as the position of invasive devices. This information is useful for confirming or changing a diagnosis, predicting prognosis, and recognizing concomitant disorders requiring therapeutic interventions, as demonstrated by our results, showing that CT scan findings led to changes in management in $26.5 \%$ of cases. An additional value of performing $\mathrm{CT}$ is represented by the evidence that intrahospital transport for CT scans can be performed relatively safely.

\section{REFERENCES}

1. ARDS Definition Task Force, Ranieri VM, Rubenfeld GD, Thompson BT, Ferguson ND, Caldwell E, et al. Acute respiratory distress syndrome: the Berlin definition. JAMA 2012;307(23):2526-2533.

2. John S, Willam C. [Lung and kidney failure: pathogenesis, interactions, and therapy]. Med Klin Intensivmed Notfmed 2015;110(6):452-458.

3. Figueroa-Casas JB, Brunner N, Dwivedi AK, Ayyappan AP. Accuracy of the chest radiograph to identify bilateral pulmonary infiltrates consistent with the diagnosis of acute respiratory distress syndrome using computed tomography as reference standard. J Crit Care 2013; 28(4):352-357.

4. Gattinoni L, Caironi P, Cressoni M, Chiumello D, Ranieri VM, Quintel M, et al. Lung recruitment in patients with the acute respiratory distress syndrome. N Engl J Med 2006;354(17):1775-1786.

5. Goodman LR, Fumagalli R, Tagliabue P, Tagliabue M, Ferrario M, Gattinoni L, Pesenti A. Adult respiratory distress syndrome due to pulmonary and extrapulmonary causes: CT, clinical, and functional correlations. Radiology 1999;213(2):545-552.

6. Chung JH, Kradin RL, Greene RE, Shepard JA, Digumarthy SR. CT predictors of mortality in pathology confirmed ARDS. Eur Radiol 2011;21(4):730-737.

7. Grieser C, Goldmann A, Steffen IG, Kastrup M, Fernández CM, Engert $\mathrm{U}$, et al. Computed tomography findings from patients with ARDS due to Influenza A (H1N1) virus-associated pneumonia. Eur J Radiol 2012;81(2):389-394.

8. Burnham EL, Hyzy RC, Paine R 3rd, Kelly AM, Quint LE, Lynch D, et al. Detection of fibroproliferation by chest high-resolution CT scan in resolving ARDS. Chest 2014;146(5):1196-1204.

9. Wilcox ME, Patsios D, Murphy G, Kudlow P, Paul N, Tansey CM, et al. Radiologic outcomes at 5 years after severe ARDS. Chest 2013;143(4):920-926.
10. Parmentier-Decrucq E, Poissy J, Favory R, Nseir S, Onimus T, Guerry $\mathrm{MJ}$, et al. Adverse events during intrahospital transport of critically ill patients: incidence and risk factors. Ann Intensive Care 2013; 3(1): 10 .

11. Schwebel C, Clec'h C, Magne S, Minet C, Garrouste-Orgeas M, Bonadona A, et al. Safety of intrahospital transport in ventilated critically ill patients: a multicenter cohort study. Crit Care Med 2013;41(8):1919-1928.

12. Tagliabue M, Casella TC, Zincone GE, Fumagalli R, Salvini E. CT and chest radiography in the evaluation of adult respiratory distress syndrome. Acta Radiol 1994;35(3):230-234.

13. Miller WT Jr, Tino G, Friedburg JS. Thoracic CT in the intensive care unit: assessment of clinical usefulness. Radiology 1998;209(2): 491-498.

14. Nierhaus A, de Heer G, Kluge S. Concept for a department of intensive care. Med Klin Intensivmed Notfmed 2014;109(7):509-515.

15. Hansell DM, Bankier AA, MacMahon H, McLoud TC, Müller NL, Remy J. Fleischner Society: glossary of terms for thoracic imaging. Radiology 2008;246(3):697-722.

16. Ichikado K, Suga M, Muranaka H, Gushima Y, Miyakawa H, Tsubamoto $\mathrm{M}$, et al. Prediction of prognosis for acute respiratory distress syndrome with thin-section CT: validation in 44 cases. Radiology 2006;238(2):321-329.

17. Hazlinger M, Ctvrtlik F, Langova K, Herman M. Quantification of pleural effusion on CT by simple measurement. Biomed Pap Med Fac Univ Palacky Olomouc Czech Repub 2014;158(1):107-111.

18. Wolfman NT, Myers WS, Glauser SJ, Meredith JW, Chen MY. Validity of CT classification on management of occult pneumothorax: a prospective study. AJR 1998;171(5):1317-1320.

19. Devaraj A, Hansell DM. Computed tomography signs of pulmonary hypertension: old and new observations. Clin Radiol 2009;64(8): 751-760.

20. Aliaga M, Forel JM, De Bourmont S, Jung B, Thomas G, Mahul M, et al. Diagnostic yield and safety of CT scans in ICU. Intensive Care Med 2015;41(3):436-443.

21. Fanara B, Manzon C, Barbot O, Desmettre T, Capellier G. Recommendations for the intra-hospital transport of critically ill patients. Crit Care 2010;14(3):R87.

22. Żyłkowska J, Kurzyna M, Florczyk M, Burakowska B, Grzegorczyk F, Burakowski J, et al. Pulmonary artery dilatation correlates with the risk of unexpected death in chronic arterial or thromboembolic pulmonary hypertension. Chest 2012;142(6):1406-1416.

23. Nambu A, Ozawa K, Kobayashi N, Tago M. Imaging of communityacquired pneumonia: Roles of imaging examinations, imaging diagnosis of specific pathogens and discrimination from noninfectious diseases. World J Radiol 2014;6(10):779-793.

24. Bellani G, Mauri T, Pesenti A. Imaging in acute lung injury and acute respiratory distress syndrome. Curr Opin Crit Care 2012;18(1): 29-34.

25. Chiumello D, Froio S, Bouhemad B, Camporota L, Coppola S. Clinical review: lung imaging in acute respiratory distress syndrome patients: an update. Crit Care 2013;17(6):243.

26. Zompatori M, Ciccarese F, Fasano L. Overview of current lung imaging in acute respiratory distress syndrome. Eur Respir Rev 2014; 23(134):519-530.

27. Lichtenstein DA. Lung ultrasound in the critically ill. Ann Intensive Care 2014;4(1):1.

28. Corradi F, Brusasco C, Pelosi P. Chest ultrasound in acute respiratory distress syndrome. Curr Opin Crit Care 2014;20(1):98-103. 